\title{
Quantum Dynamics of Photoinduced Electron-Transfer Reactions in Dye-Semiconductor Systems: First-Principles Description and Application to Coumarin 343- $\mathrm{TiO}_{2}$
}

\author{
Ivan Kondov, ${ }^{\dagger, \|}$ Martin Čížek, Claudia Benesch, ${ }^{\dagger}$ Haobin Wang, ${ }^{*, \S}$ and Michael Thoss ${ }^{*, \dagger}$ \\ Department of Chemistry, Technical University of Munich, Lichtenbergstrasse 4, D-85747 Garching, Germany, \\ Institute of Theoretical Physics, Faculty of Mathematics and Physics, Charles University, Prague, \\ Czech Republic, and Department of Chemistry and Biochemistry, MSC 3C, New Mexico State University, \\ Las Cruces, New Mexico 88003
}

Received: March 20, 2007; In Final Form: May 25, 2007

\begin{abstract}
A method to describe the quantum dynamics of photoinduced heterogeneous electron-transfer processes at dye-semiconductor interfaces is proposed. The method is based on a model Hamiltonian, the parameters of which are determined by first-principles electronic structure calculations and a partitioning scheme to define localized donor and acceptor states as well as donor-acceptor coupling matrix elements. On the basis of this modeling procedure, accurate quantum dynamical simulations are performed employing the multilayer multiconfiguration time-dependent Hartree method. As a representative example, applications to coumarin 343 adsorbed on titanium oxide nanoparticles are presented. The results of the simulations show that the ultrafast electron-injection process in this system is accompanied by electronic coherence effects, which are partially quenched due to electronic-nuclear coupling.
\end{abstract}

\section{Introduction}

Photoinduced electron-transfer (ET) reactions at dyesemiconductor interfaces represent an interesting class of ET processes. In particular, the process of electron injection from an electronically excited state of a dye molecule into a semiconductor substrate has been investigated in great detail experimentally in recent years. ${ }^{1-17}$ This process represents a key step for photonic energy conversion in nanocrystalline solar cells. ${ }^{2,6,9,18,19}$ Employing femtosecond spectroscopy techniques, it has been demonstrated that electron-injection processes at dye-semiconductor interfaces often take place on an ultrafast (sub-picosecond) time scale. $3,8,9,11,13,14,20$ For example, electroninjection times as fast as $6 \mathrm{fs}$ have been reported for alizarin adsorbed on $\mathrm{TiO}_{2}$ nanoparticles ${ }^{14}$ in time-resolved experiments, and even faster ET times have been found for biisonicotinic acid on a $\mathrm{TiO}_{2}$ surface employing resonant photoemission spectroscopy. ${ }^{11}$ Other interesting aspects of these ultrafast interfacial ET reactions are the nonequilibrium character and the influence of electronic-nuclear coupling. For example, studies of dye-semiconductor systems with electron-injection time scales on the order of a few tens to a few hundred femtoseconds indicate that the coupling of the electronic dynamics to the nuclear (i.e., vibrational) motion of the chromophore may have a significant impact on the injection process. ${ }^{8,20}$ As a result of this correlated electronic-nuclear dynamics, the electron injection in these systems is often not a simple exponential decay process and thus cannot be characterized by a single rate constant.

The theoretical study of such interfacial ET processes requires a quantum mechanical description of the electron-injection

* Authors to whom correspondence should be addressed. E-mail: whb@intrepid.nmsu.edu (H.W.) and michael.thoss@ch.tum.de (M.T.).

$\dagger$ Technical University of Munich.

$\doteqdot$ Charles University.

$\S$ New Mexico State University.

" Present address: Institute for Scientific Computing, Forschungszentrum Karlsruhe, P.O. Box 3640, 76021 Karlsruhe, Germany. dynamics including the coupling to the nuclear degrees of freedom. Since it is currently not feasible to carry out a full quantum dynamical simulation of the interfacial ET dynamics that takes into account the coupling to the nuclear degrees of freedom and employs an adequate electronic structure theory, different approximate strategies have been applied. One possibility is to use a first-principles electronic structure method to describe the dye-semiconductor system but employ an approximate treatment of the nuclear motion. An example is $\mathrm{ab}$ initio molecular dynamics, where the dynamics of the nuclear degrees of freedom are described classically. ${ }^{21-26}$ Another strategy, which is particularly useful if an accurate, fully quantum dynamical treatment is required, is to use a physically motivated model. A commonly used model to study heterogeneous ET processes at interfaces is the Anderson-Newns model. ${ }^{27}$ Along this line, several workers have studied the electron-injection dynamics based on models of reduced dimensionality, taking into account typically a single reaction mode. ${ }^{17,28-36}$ Dissipative effects, such as vibrational relaxation of the reaction mode, have been considered (for weak coupling) within Redfield theory. ${ }^{31}$ In a recent model study, we have investigated in detail the influence of multidimensional coherent and dissipative vibrational motion on the electron-injection dynamics, ${ }^{37}$ employing the self-consistent hybrid approach ${ }^{38,39}$ in combination with the multilayer multiconfiguration timedependent Hartree method. ${ }^{40}$ These methods allow an accurate quantum dynamical description of the ET process beyond the limitations of perturbation theory.

To apply such models to experimentally studied systems, various model parameters such as the energies and couplings of the relevant electronic states, the important vibrational modes of the chromophore, and the electronic-vibrational couplings need to be determined. If only a single (or a few) reaction mode(s) is considered and a simple model for the donoracceptor coupling (e.g., the wide-band approximation) is used, then it is possible to determine the parameters empirically based 
on experimental spectra. ${ }^{17}$ To simulate interfacial ET dynamics in systems with multidimensional nuclear dynamics, we have recently employed a semiempirical strategy, where the vibrational modes and electronic-vibrational couplings were determined by electronic structure calculations, ${ }^{41,42}$ while for the donor-acceptor coupling and the description of the semiconductor substrate an empirically parametrized tight-binding model was utilized. ${ }^{43,41}$ As a step toward a fully first-principles-based description of quantum dynamics in dye-semiconductor systems, we propose here a method to determine the electronic energies and donor-acceptor coupling matrix elements from electronic structure calculations.

The determination of donor-acceptor couplings in ET reactions requires, in principle, the definition of charge-localized diabatic states. To this end, several different approaches have been proposed, including the Mulliken-Hush method ${ }^{44,45}$ and its generalization ${ }^{46,47}$ as well as the fragment charge difference approach. ${ }^{48}$ Besides these methods, which are specific to the ET problem, there exist a variety of general diabatization schemes. ${ }^{49}$ The introduction of charge-localized diabatic states for dye-semiconductor systems involves the additional complication that a (quasi-)continuum of acceptor states (corresponding to the (quasi-)conduction band of the semiconductor nanoparticle or surface) has to be treated. Thus, the problem involves the treatment of a single (or a small set of) discrete donor state(s), which is (are) embedded in a (quasi-)continuum of acceptor states. This problem is closely related to the treatment of resonances in scattering theory, e.g., electronic resonance states in low-energy electron-molecule scattering. In this context, the projection-operator approach ${ }^{50}$ has been proven to be a very useful concept to introduce localized diabatic states. ${ }^{51-53}$ For the present problem, we employ a method that is similar to the projection-operator approach. The method is based on a partitioning of the overall Hilbert space into a donor and acceptor part and a subsequent separate diagonalization of the two blocks of the Hamiltonian to define the donor and acceptor states. As a first step, in the present paper we work within the mean-field single-electron picture and use atomic orbitals to introduce the partitioning. A treatment within correlated many-electron states will be the subject of future work.

\section{Theory}

In this section, the theoretical methodology used to describe photoinduced ET reactions in dye-semiconductor systems is outlined. The major focus is thereby on the method to determine electronic energies and donor-acceptor coupling matrix elements. To keep the paper self-contained, we also discuss briefly the method used to describe the nuclear degrees of freedom as well as the dynamical approach employed in the simulation.

A. Electron-Transfer Hamiltonian. To study ET dynamics in dye-semiconductor systems, we use an ab initio (firstprinciples)-based model for heterogeneous ET reactions. Within this model the Hamiltonian is represented in a basis of the following diabatic (charge-localized) electronic states that are relevant for the photoreaction: the electronic ground state of the overall system $\left|\psi_{g}\right\rangle$, the donor state of the ET process $\left|\psi_{d}\right\rangle$ (which, in the limit of vanishing coupling between chromophore and semiconductor substrate, corresponds to the product of an electronically excited state of the chromophore and an empty conduction band of the semiconductor), and the (quasi-)continuum of acceptor states of the ET reaction $\left|\psi_{k}\right\rangle$ (corresponding in the zero coupling limit to the product of the cationic state of the chromophore and a conduction band state of the semiconductor substrate). Thus, the Hamiltonian reads

$$
\begin{array}{r}
H_{s}=T+\left|\psi_{g}\right\rangle V_{g}\left\langle\psi_{g}|+| \psi_{d}\right\rangle V_{d d}\left\langle\psi_{d}\left|+\sum_{k}\right| \psi_{k}\right\rangle V_{k k}\left\langle\psi_{k}\right|+ \\
\sum_{k}\left(\left|\psi_{d}\right\rangle V_{d k}\left\langle\psi_{k}|+| \psi_{k}\right\rangle V_{k d}\left\langle\psi_{d}\right|\right)
\end{array}
$$

with the kinetic energy of the nuclei (we use mass-scaled coordinates and atomic units throughout the paper)

$$
T=\frac{1}{2} \sum_{l} P_{l}^{2}
$$

the potential energy in the electronic ground state, $V_{g}(Q)$, and the diabatic (donor-acceptor) potential matrix $V_{i j}(Q)$, which depend on the nuclear coordinates $Q$. The diagonal elements of the diabatic potential matrix, $V_{d d}$ and $V_{k k}$, describe the energies of the electronic donor and acceptor states, respectively, while the nondiagonal elements, $V_{d k}$, characterize the donor-acceptor ET coupling.

The potential energy in the electronic ground state, $V_{g}$, can (at least in principle) rather straightforwardly be determined with electronic structure calculations. As discussed in the introduction, the characterization of the diabatic (donor-acceptor) potential matrix $V_{i j}$, however, requires the introduction of suitable diabatic donor and acceptor states, $\left|\psi_{d}\right\rangle$ and $\left|\psi_{k}\right\rangle$.

B. Determination of Electronic Energies and DonorAcceptor Coupling Matrix Elements. In our previous work on heterogeneous ET, ${ }^{37,41}$ we have used a semiempirical method, motivated by the Newns model of chemisorption, ${ }^{27}$ with a parametrization based on a tight-binding model, ${ }^{43}$ to characterize the diabatic states $\left|\psi_{d}\right\rangle$ and $\left|\psi_{k}\right\rangle$ and thus to determine the electronic energies and donor-acceptor coupling matrix elements $V_{d k}$. In the following, we will refer to it as the semiempirical Newns model.

As a first step toward a first-principles description of quantum dynamics in dye-semiconductor systems, we use here an approach based on electronic structure calculations. This approach is motivated by the projection-operator approach of resonant electron-molecule scattering. ${ }^{53}$ Specifically, we employ a partitioning scheme based on density functional theory (DFT) calculations for a complex of the dye molecule with a finite $\mathrm{TiO}_{2}$ cluster. The scheme for defining the diabatic states $\left|\psi_{d}\right\rangle$ and $\left|\psi_{k}\right\rangle$ in the Hamiltonian (eq 2.1) is based on three steps: (i) a partitioning of the Hilbert space in a donor and acceptor group using a localized basis, (ii) a partitioning of the Hamiltonian according to the donor-acceptor separation, and (iii) a separate diagonalization of the donor and acceptor blocks of the partitioned Hamiltonian. ${ }^{54}$ In the present paper, we work within the mean-field single-electron picture. Thus we identify the effective Hamiltonian with the Fock (or Kohn-Sham) matrix and use the orbitals and orbital energies in the partitioning method.

Various types of localized basis functions can be used to separate the donor and acceptor space. Here, we employ the atomic orbitals $\left|\varphi_{j}\right\rangle$ used in the electronic structure calculation for the overall system. The atomic orbitals are based on Gaussian functions, which are well localized in space. The set of atomic orbitals of the overall system, $\left|\varphi_{j}\right\rangle$, is divided into two groupsthe donor group $\left(\left|\varphi_{j}^{d}\right\rangle\right)$, which comprises the orbitals centered at the atoms of the dye molecule, and the acceptor group $\left(\left|\varphi_{j}^{a}\right\rangle\right)$, which includes the orbitals centered at the $\mathrm{TiO}_{2}$ cluster.

Since it is advantageous to work with orthogonal orbitals, ${ }^{55,56}$ the set of atomic orbitals of the overall system is orthogonalized according to Löwdin 57,58 


$$
\left|\tilde{\varphi}_{n}\right\rangle=\sum_{j}\left(S^{-1 / 2}\right)_{j n}\left|\varphi_{j}\right\rangle
$$

where $S$ denotes the atomic orbital overlap matrix with elements $S_{k l}=\left\langle\varphi_{k} \mid \varphi_{l}\right\rangle$. The new basis functions obtained, $\left|\tilde{\varphi}_{n}\right\rangle$, exhibit a minimal deviation from the original ones in a least-square sense, and hence, their localization is preserved. In particular, the classification as donor $\left(\left|\tilde{\varphi}_{n}^{d}\right\rangle\right)$ or acceptor $\left(\left|\tilde{\varphi}_{n}^{a}\right\rangle\right)$ orbitals is still valid. In contrast to other orthogonalization procedures, such as the Gram-Schmidt method, the symmetric orthogonalization according to Löwdin has also the advantage that it is "least biased".

The new set of orthogonal basis functions is then used to partition the Fock (or Kohn-Sham) matrix from the converged SCF (DFT) calculation into the two (donor and acceptor) subspaces. The Fock matrix in the orthogonal basis is given by

$$
\tilde{F}=S^{-1 / 2} F S^{-1 / 2}
$$

where $F$ denotes the Fock matrix in the original atomic orbital basis. The Fock matrix can be arranged in the following donoracceptor block structure

$$
\tilde{F}=\left(\begin{array}{ll}
\tilde{F}_{a d} & \tilde{F}_{d a} \\
\tilde{F}_{a d} & \tilde{F}_{a a}
\end{array}\right)
$$

where the matrix elements are given by

$$
\begin{aligned}
\tilde{F}_{\alpha \beta, n m} & =\left\langle\tilde{\varphi}_{n}^{\alpha}|f| \tilde{\varphi}_{m}^{\beta}\right\rangle \\
& =\sum_{i}\left\langle\tilde{\varphi}_{n}^{\alpha} \mid \chi_{i}\right\rangle \epsilon_{i}\left\langle\chi_{i} \mid \tilde{\varphi}_{m}^{\beta}\right\rangle
\end{aligned}
$$

Here, $f$ is the Fock operator, $\left|\chi_{i}\right\rangle$ denote the molecular orbitals resulting from the SCF calculation, i.e., $f\left|\chi_{i}\right\rangle=\epsilon_{i}\left|\chi_{i}\right\rangle$, and the Greek indices $\alpha$ and $\beta$ denote either the donor (" $d$ ") or the acceptor (" $a$ ") subspace.

Separate diagonalization of the two (donor and acceptor) blocks of the Fock matrix $\tilde{F}_{\alpha \alpha}$ via

$$
\bar{F}_{\alpha \alpha}=D_{\alpha}^{\dagger} \tilde{F}_{\alpha \alpha} D_{\alpha}
$$

and transformation of the off-diagonal parts to the corresponding eigenstates in the two blocks

$$
\bar{F}_{\alpha \beta}=D_{\alpha}^{\dagger} \tilde{F}_{\alpha \beta} D_{\beta}
$$

result in the following prediagonalized block structure

$$
\bar{F}=\left(\begin{array}{ll}
\bar{F}_{d d} & \bar{F}_{d a} \\
\bar{F}_{a d} & \bar{F}_{a a}
\end{array}\right)=\left(\begin{array}{llllll}
\epsilon_{d, 1} & 0 & \ldots & & & \\
0 & \epsilon_{d, 2} & \ldots & & \bar{F}_{d a} & \\
\vdots & \vdots & & & & \\
& & & \epsilon_{a, 1} & 0 & \ldots \\
& \overline{\mathrm{F}}_{a d} & 0 & \epsilon_{a, 2} & \ldots \\
& & & \vdots & \vdots &
\end{array}\right)
$$

The corresponding donor and acceptor molecular orbitals, $\left|\bar{\varphi}_{n}^{\alpha}\right\rangle$, are given as the eigenvectors of $\tilde{F}_{\alpha \alpha}$ and are related to the orthogonalized atomic orbitals $\left|\tilde{\varphi}_{j}^{\alpha}\right\rangle$ and the original atomic orbitals $\left|\varphi_{l}\right\rangle$ via

$$
\begin{aligned}
\left|\bar{\varphi}_{n}^{\alpha}\right\rangle & =\sum_{j}\left(D_{\alpha}\right)_{j n}\left|\tilde{\varphi}_{j}^{\alpha}\right\rangle \\
& =\sum_{j, l}\left(D_{\alpha}\right)_{j n}\left(S^{-1 / 2}\right)_{l j}\left|\varphi_{l}\right\rangle
\end{aligned}
$$

The diagonal blocks of the Fock matrix (eq 2.9) with indices $d$ and $a$ contain the energies of the localized chromophore states and those of the cluster, respectively. The off-diagonal blocks contain the electronic coupling elements between chromophore and cluster sites. Identifying the donor state $\left|\psi_{d}\right\rangle$ with one of the states $\left|\bar{\varphi}_{n}^{d}\right\rangle$ (based, e.g., on the orbital energy or the transition dipole moment to the ground state) and the acceptor states $\left|\psi_{k}\right\rangle$ with the states $\left|\bar{\varphi}_{k}^{a}\right\rangle$, the electronic energies and the donor acceptor matrix elements are given by $V_{d d}=\epsilon_{d, n}, V_{k k}=$ $\epsilon_{a, k}$, and $V_{d k}=\bar{F}_{d a, n k}$, respectively. It is obvious that this method can also be used in cases where several donor states are involved in the ET reaction.

The partitioning method discussed above is not limited to dye-semiconductor systems with a finite semiconductor cluster but can, in principle, also be applied to a dye molecule adsorbed on an extended surface. One possibility is to employ a slab model and electronic structure calculations with periodic boundary conditions. Alternatively, the effect of an infinite semiconductor substrate can also be described using surface Green's function techniques. ${ }^{59}$ Within this method, the effect of the infinite substrate enters via the self-energy. In the application considered below, we have used a simpler approximate version of this method to mimic the effect of an extended surface. Thereby a constant imaginary part is added to the atomic orbital energies (in the orthogonal basis $\left|\tilde{\varphi}_{j}^{a}\right\rangle$ ) at the outer atoms of the $\mathrm{TiO}_{2}$ cluster. The details of this method are described in the Appendix.

C. Characterization of Nuclear Degrees of Freedom. To characterize the nuclear degrees of freedom, the partitioning procedure outlined above has to be performed for each nuclear geometry, thus resulting in diabatic potential energy surfaces $V_{i i}(Q)$ and coordinate-dependent donor-acceptor coupling matrix elements $V_{d k}(Q)$. If many nuclear degrees of freedom are important, as in the system considered below, such a global characterization of the potential energy surfaces is not feasible. A more practical, local procedure is to employ the normal modes $(Q)$ of the electronic ground state and expand the diabatic potential matrix elements $V_{i j}(Q)$ around the equilibrium geometry of the ground state of the overall system. This approach is appropriate for photoinduced ultrafast dynamics in systems without large amplitude motion. It has been applied recently for the related problem of molecular conduction through a metal-molecule-metal junction. ${ }^{60}$

For the present purpose of testing the partitioning procedure, we adopt the strategy used in our previous work, where the vibrational parameters and electronic-vibrational coupling parameters are determined based on electronic structure calculations for the isolated chromophore, thereby neglecting the coupling to the semiconductor substrate and to the phonons of the semiconductor. The extension of the method to include this interaction will be presented in a future publication.

The approach has been described in detail elsewhere. ${ }^{41}$ Briefly, we perform a vibrational analysis of the isolated chromophore in the electronic ground state and employ the harmonic approximation for the corresponding potential energy surface

$$
V_{g}(Q)=\epsilon_{g}+\frac{1}{2} \sum_{l} \Omega_{l}{ }^{2} Q_{l}^{2}
$$

Here, $Q_{l}$ denotes the $l$ th normal mode (with frequency $\Omega_{l}$ ), and $\epsilon_{g}$ is the ground-state equilibrium energy. The latter is obtained from an electronic structure calculation for the overall (dyesemiconductor) system. We assume the donor-acceptor cou- 
pling matrix elements $V_{d k}$ to be approximately independent of the nuclear geometry ${ }^{61}$ and expand the diabatic potential energy surfaces around the equilibrium geometry of the electronic ground state, $Q_{0}$

$$
V_{j j}(Q)=V_{j j}\left(Q_{0}\right)+\sum_{l} \kappa_{l}^{j} Q_{l}+\sum_{l, k} \gamma_{l k}^{j} Q_{l} Q_{k}
$$

In the simplest approximation, only the linear term of the expansion is taken into account. The frequencies are approximated by their ground-state values, and Dushinsky rotation $^{62}$ of the normal modes is neglected. In this way we obtain

$$
V_{j j}(Q)=V_{j j}\left(Q_{0}\right)+\sum_{l} \kappa_{l}^{j} Q_{l}+\frac{1}{2} \sum_{l} \Omega_{l}^{2} Q_{l}^{2}
$$

This approximation has been used successfully to describe Franck-Condon and resonance Raman spectra. ${ }^{63}$ It is also used in the linear vibronic coupling model of conical intersections ${ }^{64}$ and in the Marcus theory of ET. ${ }^{65}$

Within the description of the nuclear degrees of freedom employed here, the parameters of the diabatic potential energy surfaces of the donor and acceptor states are obtained from the potential energy functions of the excited state of the neutral chromophore and the ground state of the cation of the chromophore, respectively. Accordingly, we have

$$
\begin{aligned}
& V_{d d}(Q)=\epsilon_{d}+\sum_{l} \kappa_{l}^{d} Q_{l}+\frac{1}{2} \sum_{l} \Omega_{l}^{2} Q_{l}^{2} \\
& V_{k k}(Q)=\epsilon_{k}+\sum_{l} \kappa_{l}^{a} Q_{l}+\frac{1}{2} \sum_{l} \Omega_{l}^{2} Q_{l}^{2}
\end{aligned}
$$

where $\epsilon_{d}$ and $\epsilon_{k}$ denote the energy of the donor and acceptor states (at the equilibrium geometry of the ground state), respectively, which are obtained from an electronic structure calculation of the overall system. The electronic-vibrational coupling constants, $\kappa_{l}^{d}$ and $\kappa_{l}^{a}$, are obtained from the gradients of the excited state of the neutral chromophore (corresponding to the donor state) and the ground state of the cation of the chromophore (corresponding to the acceptor state) at the equilibrium geometry of the ground state of the neutral chromophore. The details of the electronic structure calculations as well as the specific parameters for the system considered below (coumarin 343 at titanium oxide) are described elsewhere. ${ }^{41,42}$

In most experiments on electron injection in dye-semiconductor systems, a colloidal solution of dye-sensitized nanoparticles was employed. ${ }^{3,5,6,13}$ To account for the influence of the surrounding solvent on the ET dynamics in our simulations, we employ a standard (outer-sphere) linear response model ${ }^{66-68}$ where the Hamiltonian of the dye-semiconductor system is coupled linearly to a bath of harmonic oscillators. Thus the Hamiltonian of the overall system reads

$$
H=H_{s}+H_{b}+H_{s b}
$$

with $H_{s}$ given by eq 2.1 and

$$
\begin{gathered}
H_{b}=\frac{1}{2} \sum_{j}\left(p_{j}^{2}+\omega_{j}^{2} x_{j}^{2}\right) \\
H_{s b}=\left|\psi_{d}\right\rangle \sum_{j} c_{j}^{d} x_{j}\left\langle\psi_{d}\left|+\sum_{k}\right| \psi_{k}\right\rangle \sum_{j} c_{j}^{a} x_{j}\left\langle\psi_{k}\right|
\end{gathered}
$$

The parameters of the solvent part of the Hamiltonian are characterized by the spectral densities

$$
\begin{aligned}
& J^{d}(\omega)=\frac{\pi}{2} \sum_{j} \frac{\left(c_{j}^{d}\right)^{2}}{\omega_{j}} \delta\left(\omega-\omega_{j}\right) \\
& J^{a}(\omega)=\frac{\pi}{2} \sum_{j} \frac{\left(c_{j}^{a}\right)^{2}}{\omega_{j}} \delta\left(\omega-\omega_{j}\right)
\end{aligned}
$$

in the donor and acceptor states, respectively. The spectral densities describe the response of the solvent polarization to the change of the charge distribution of the solute associated with electronic transitions from the ground electronic state to the excited state of the chromophore and to the cation, respectively. In principle, the spectral densities can be different for the two electronic transitions. Here we use for simplicity a modeling where the response of the solvent for both transitions is described by a coupling to the same bath that differs only in the overall coupling strength, i.e., $c_{j}^{a}=\alpha c_{j}^{d}$. (Accordingly, in the following the superscript for the coupling constants will be omitted, i.e., $c_{j} \equiv c_{j}^{d}$.) It is emphasized that this approximation is only invoked for the solvent bath modes but not for the intramolecular modes. This description is in accordance with simple dielectric continuum theories of relaxation in polar solvents. ${ }^{41,68-70}$ As a result of this assumption, we have

$$
J_{b}^{a}(\omega)=\alpha^{2} J_{b}^{d}(\omega)=\alpha^{2} \frac{\pi}{2} \sum_{j} \frac{c_{j}^{2}}{\omega_{j}} \delta\left(\omega-\omega_{j}\right)
$$

The solvent reorganization energies associated with the transitions from the electronic ground to the excited state and to the cation of the chromophore are given by

$$
\begin{gathered}
\lambda^{d}=\sum_{j} \frac{\left(c_{j}^{d}\right)^{2}}{2 \omega_{j}^{2}} \equiv \sum_{j} \frac{c_{j}^{2}}{2 \omega_{j}^{2}} \\
\lambda^{a}=\sum_{j} \frac{\left(c_{j}^{a}\right)^{2}}{2 \omega_{j}^{2}}=\alpha^{2} \sum_{j} \frac{c_{j}^{2}}{2 \omega_{j}^{2}}
\end{gathered}
$$

The solvent reorganization energy for the ET process, which corresponds to a transition from the electronically excited state to the cation of the chromophore, however, is given by

$$
\lambda^{\mathrm{ET}}=\sum_{j} \frac{\left(c_{j}^{d}-c_{j}^{a}\right)^{2}}{2 \omega_{j}^{2}}=(1-\alpha)^{2} \sum_{j} \frac{c_{j}^{2}}{2 \omega_{j}^{2}}
$$

As has been discussed in detail in ref 41, the spectral densities of the solvent model are chosen to be of bimodal form

$$
\begin{gathered}
J^{d}(\omega)=\sqrt{\pi} \frac{\lambda_{G} \omega}{\omega_{G}} \mathrm{e}^{-\left[\omega /\left(2 \omega_{\mathrm{G}}\right)\right]^{2}}+2 \lambda_{\mathrm{D}} \frac{\omega \omega_{\mathrm{D}}}{\omega^{2}+\omega_{\mathrm{D}}^{2}} \\
J^{a}(\omega)=\alpha^{2} J^{d}(\omega)
\end{gathered}
$$

with a Gaussian part accounting for the ultrafast inertial dynamics of the solvent polarization and a Debye part describing the slower diffusive decay. The corresponding parameters, $\omega_{\mathrm{G}}$ $=144 \mathrm{~cm}^{-1}, \omega_{\mathrm{D}}=25 \mathrm{~cm}^{-1}, \lambda_{\mathrm{D}}^{d}=\lambda_{\mathrm{G}}^{d}=700 \mathrm{~cm}^{-1}$, and $\alpha=$ -0.1 , have been chosen in accordance with experimental results 
on solvation dynamics and absorption spectra of coumarin 343 in water. ${ }^{13,71}$

D. Observables of Interest and Dynamical Methods. Several observables are of interest for the study of heterogeneous ET in dye-semiconductor systems, in particular, the electronic population dynamics, the associated nuclear wave packet dynamics, as well as stationary and time-resolved spectra. In this work, we will concentrate on the photoinduced electronic injection dynamics, which are most directly reflected by the time-dependent population of the donor state

$$
\begin{aligned}
P_{d}(t)= & \frac{1}{\operatorname{Tr}\left[\mathrm{e}^{-\beta H_{\mathrm{Ng}}}\right]} \operatorname{Tr}\left[\mathrm{e}^{-\beta H_{\mathrm{Ng}}}\left|\psi_{d}\right\rangle\left\langle\psi_{d}\left|\mathrm{e}^{\mathrm{i} H t}\right| \psi_{d}\right\rangle\left\langle\psi_{d}\right| \mathrm{e}^{-\mathrm{i} H t}\right]
\end{aligned}
$$

Here, we have assumed that the system is initially prepared by an ultrafast laser pulse in the donor state $\left|\psi_{d}\right\rangle$. The initial state of the nuclear degrees of freedom is specified by the Boltzmann operator $\mathrm{e}^{-\beta H_{\mathrm{Ng}}}$ of the nuclear Hamiltonian in the electronic ground state

$$
H_{\mathrm{Ng}}=\frac{1}{2} \sum_{l}\left(P_{l}^{2}+\Omega_{l}^{2} Q_{l}^{2}\right)+\frac{1}{2} \sum_{j}\left(p_{j}^{2}+\omega_{j}^{2} x_{j}^{2}\right)
$$

To simulate the quantum dynamics of this system, we use the multilayer (ML) formulation ${ }^{40,72}$ of the multiconfiguration time-dependent Hartree (MCTDH) method ${ }^{73-76}$ in combination with an importance sampling scheme to describe the thermal initial conditions in the observables introduced above. The method as well as applications to different reactions in the condensed phase have been described in detail previously. ${ }^{37,40,77}$ Here, we only briefly introduce the general idea and give some details specific to the application in this work.

The ML-MCTDH method ${ }^{40}$ is a variational approach for the description of quantum dynamics in systems with many degrees of freedom. It extends the original MCTDH method ${ }^{73-76}$ for application to significantly larger systems. In the original (singlelayer) MCTDH method, the overall wave function is expanded in terms of time-dependent configurations

$$
\begin{aligned}
&|\Psi(t)\rangle=\sum_{J} A_{J}(t)\left|\Phi_{J}(t)\right\rangle \equiv \\
& \sum_{j_{1}} \sum_{j_{2}} \cdots \sum_{j_{M}} A_{j_{1} j_{2} \cdots j_{M}}(t) \prod_{k=1}^{M}\left|\phi_{j k}^{k}(t)\right\rangle
\end{aligned}
$$

Here, $\left|\phi_{j k}^{k}(t)\right\rangle$ is the "single-particle" (SP) function for the $k$ th SP degree of freedom, and $M$ denotes the number of SP degrees of freedom. Each SP group usually contains several (Cartesian) degrees of freedom in our calculation, and for convenience the SP functions within the same SP degree of freedom are chosen to be orthonormal.

In contrast to the original MCTDH method, where the SP functions are represented by time-independent basis functions

$$
\left|\phi_{n}^{k}(t)\right\rangle=\sum_{I} B_{I}^{k, n}(t)\left|u_{I}^{k}\right\rangle
$$

the ML-MCTDH method employs a dynamic contraction of the basis functions that constitute the SP functions. To this end, a time-dependent multiconfigurational expansion of the SP functions is used

$$
\begin{aligned}
\left|\phi_{n}^{k}(t)\right\rangle=\sum_{I} B_{I}^{k, n}(t)\left|u_{I}^{k}(t)\right\rangle \equiv \\
\sum_{i_{1}} \sum_{i_{2}} \cdots \sum_{i_{Q(k)}} B_{i_{1} i_{2} \cdots i_{Q(k)}}^{k, n}(t) \prod_{q=1}^{Q(k)}\left|v_{i_{q}}^{k, q}(t)\right\rangle
\end{aligned}
$$

i.e., the basic strategy of MCTDH is adopted to treat each SP function. Here, $Q(k)$ denotes the number of level two (L2) SP degrees of freedom in the $k$ th level one (L1) SP group, and $\left|v_{i_{q}}^{k, q}(t)\right\rangle$ is the L2-SP function for the $q$ th L2-SP degree of freedom. Employing two dynamical layers, the expansion of the overall wave function can thus be written in the form

$$
\begin{gathered}
|\Psi(t)\rangle=\sum_{j_{1}} \sum_{j_{2}} \cdots \sum_{j_{M}} A_{j_{1} j_{2} \cdots j_{M}}(t) \\
\times \prod_{k=1}^{M}\left[\sum_{i_{1}} \sum_{i_{2}} \cdots \sum_{i_{Q(k)}} B_{i_{1} i_{2}}^{k, j_{k}} \cdots{ }_{Q(k)}(t) \prod_{q=1}^{Q(k)}\left|v_{i_{q}}^{k, q}(t)\right\rangle\right]
\end{gathered}
$$

The extension to more dynamical layers is obvious. In the calculation considered below up to three dynamical layers are employed.

The equations of motion within the ML-MCTDH approach can be obtained from the Dirac-Frenkel variational principle. ${ }^{40}$ For two layers, they are given by

$$
\begin{gathered}
i|\dot{\Psi}(t)\rangle_{\mathrm{L} 1 \text { coefficients }}=\hat{H}(t)|\Psi(t)\rangle \\
i\left|\underline{\phi}^{k}(t)\right\rangle_{\mathrm{L} 2 \text { coefficients }}= \\
{\left[1-\hat{P}^{k}(t)\right]\left[\hat{\rho}^{k}(t)\right]^{-1}\langle\hat{H}(t)\rangle^{k}\left|\phi^{k}(t)\right\rangle(2.28 \mathrm{~b})} \\
i\left|\underline{\dot{v}}^{k, q}(t)\right\rangle_{\mathrm{L} 3 \text { coefficients }}= \\
{\left[1-\hat{P}_{\mathrm{L} 2}^{k, q}(t)\right]\left[\hat{\rho}^{k, q}(t)\right]^{-1}\langle\hat{\mathscr{H}}(t)\rangle^{k, q} \mid v^{k, q}(t)}
\end{gathered}
$$

where the mean-field operators, reduced densities, and projection operators are defined in ref 40 . The equations of motion for further layers are again obvious extensions of eq 2.28. The inclusion of several dynamically optimized layers in the MLMCTDH method provides more flexibility in the variational functional, which significantly advances the capabilities of performing wave packet propagations in a complex system. This has been demonstrated by several applications to quantum dynamics in the condensed phase including many degrees of freedom. ${ }^{37,40,72,77-80}$

\section{Application to the Dye-Semiconductor System Coumarin 343- $\mathrm{TiO}_{2}$}

As a representative example of interfacial ET processes, we consider photoinduced electron injection in the dye-semiconductor system coumarin $343(\mathrm{C} 343)-\mathrm{TiO}_{2}$. This system has been investigated experimentally by a number of groups. ${ }^{3,5,6,13,81}$ Recently, we have studied the electronic injection dynamics as well as the associated nuclear wave packet dynamics in this system employing a semiempirical Newns model. ${ }^{41}$ Here, we apply the first-principles model outlined above.

A. Characterization of the System, Donor-Acceptor Separation, and Coupling Matrix Elements. To model C343 adsorbed on $\mathrm{TiO}_{2}$ nanoparticles at the atomic level, we have considered complexes of $\mathrm{C} 343$ with anatase $\mathrm{TiO}_{2}$ clusters of different sizes, as depicted in Figure 1. All interatomic distances and valence angles of the $\mathrm{TiO}_{2}$ clusters were taken from the X-ray structure of bulk anatase. ${ }^{82}$ To avoid artificial effects due to dangling bonds, the clusters were saturated by adding 

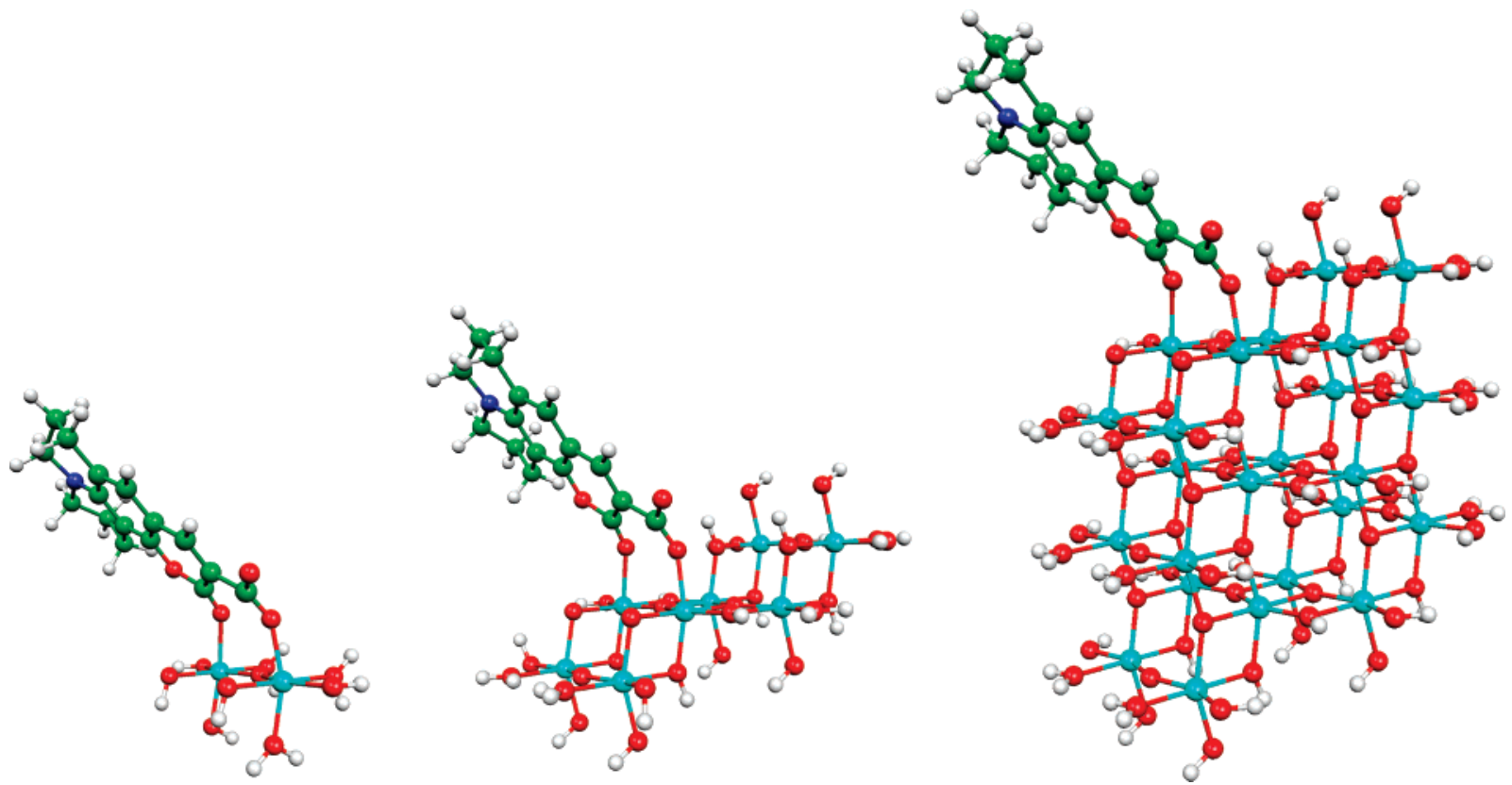

Figure 1. Complexes of $\mathrm{C} 343$ with the clusters $\left(\mathrm{TiO}_{2}\right)_{2}\left(\mathrm{H}_{2} \mathrm{O}\right)$ (left image), $\left(\mathrm{TiO}_{2}\right)_{8}\left(\mathrm{H}_{2} \mathrm{O}\right)_{14}\left(\right.$ middle image), and $\left(\mathrm{TiO}_{2}\right)_{24}\left(\mathrm{H}_{2} \mathrm{O}\right)_{30}($ right image).

hydrogen atoms and hydroxyl groups to the boundary oxygen and titanium atoms, respectively. The number of added hydrogen atoms and hydroxyl groups as well as the adsorption sites were chosen in a way to obtain neutral closed-shell clusters with high coordination of the boundary titanium and oxygen atoms. Very similar saturated cluster models have been used to study surface photoreactions on anatase nanoparticles ${ }^{83}$ as well as adsorption of water and catechol on the (101) anatase surface. ${ }^{84}$ The adsorption site of the clusters is characterized by two titanium atoms from the (101) surface of anatase (see below). The minimal model that can capture all of these features is the cluster $\left(\mathrm{TiO}_{2}\right)_{2}\left(\mathrm{H}_{2} \mathrm{O}\right)_{5}$ (Figure 1, left image). However, significantly larger clusters had to be considered to minimize boundary effects. Well converged results could be obtained with the cluster $\left(\mathrm{TiO}_{2}\right)_{24}\left(\mathrm{H}_{2} \mathrm{O}\right)_{30}$, and only these results will be reported in the following.

To obtain the structure of the $\mathrm{C} 343-\left(\mathrm{TiO}_{2}\right)_{24}\left(\mathrm{H}_{2} \mathrm{O}\right)_{30}$ complex shown in Figure 1 (right image) we have used the following protocol: The geometry of isolated C343 was taken from an earlier study. ${ }^{42}$ The deprotonated form of C343 was aligned to the saturated $\left(\mathrm{TiO}_{2}\right)_{8}\left(\mathrm{H}_{2} \mathrm{O}\right)_{14}$ cluster (Figure 1, middle image) by optimizing the ground-state energy in internal coordinates. Thereby, the internal nuclear degrees of freedom of C343 and of the cluster were kept fixed, and only the six relative orientation coordinates (three translations and three rotations) were allowed to vary. Then, $\mathrm{C} 343$ was attached to the larger $\left(\mathrm{TiO}_{2}\right)_{24}\left(\mathrm{H}_{2} \mathrm{O}\right)_{30}$ cluster (Figure 1, right image) using the six relative orientation coordinates from the alignment optimization at the smaller $\left(\mathrm{TiO}_{2}\right)_{8}\left(\mathrm{H}_{2} \mathrm{O}\right)_{14}$ cluster. The proton detached from $\mathrm{C} 343$ upon adsorption was attached to a surface oxygen atom adjacent to the adsorption center. This choice of the protocol and the adsorption site is supported by a more detailed electronic structure study on the adsorption of $\mathrm{C} 343$ on anatase $\mathrm{TiO}_{2},{ }^{85}$ which has revealed that (i) the adsorbate is deprotonated upon adsorption and the proton is attached to a doubly coordinated surface oxygen atom and (ii) the preferred complexation is bidentate bridging as shown in Figure 1 but not bidentate chelation to only one surface titanium atom.

The alignment of $\mathrm{C} 343$ relative to the cluster $\left(\mathrm{TiO}_{2}\right)_{8}\left(\mathrm{H}_{2} \mathrm{O}\right)_{14}$ was optimized with DFT using Gaussian $03^{86}$ with the B3LYP functional and the $3-21 \mathrm{G}$ basis set. All other electronic structure

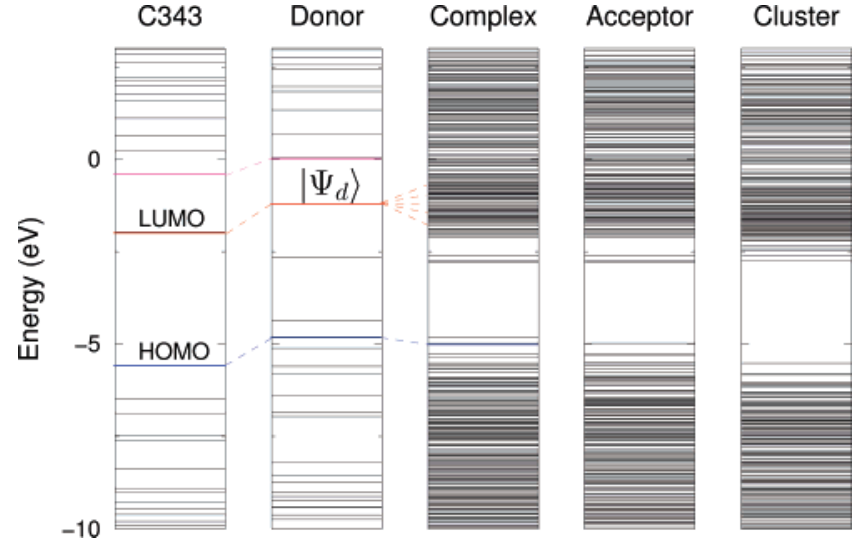

Figure 2. Representation of the energy levels of the system investigated. Shown are (from left to right) energy levels of the isolated chromophore $\mathrm{C} 343$, the donor molecular orbitals, $\left|\bar{\varphi}_{n}^{d}\right\rangle$, as obtained from the partitioning procedure, the complex $\left(\mathrm{TiO}_{2}\right)_{24}\left(\mathrm{H}_{2} \mathrm{O}\right)_{30} \mathrm{C} 343$, the acceptor molecular orbitals, $\left|\bar{\varphi}_{n}^{a}\right\rangle$, as obtained from the partitioning procedure, and the pure cluster $\left(\mathrm{TiO}_{2}\right)_{24}\left(\mathrm{H}_{2} \mathrm{O}\right)_{30}$. The orbital chosen as the donor state is denoted by $\left|\Psi_{d}\right\rangle$. Furthermore, correlations between selected energy levels are indicated.

calculations were performed with the program package TURBOMOLE $^{87}$ employing DFT with the B3LYP functional and the $\mathrm{SV}(\mathrm{P})$ basis set.

To discuss the donor-acceptor partitioning procedure, we first consider the energy-level scheme. Figure 2 shows the energies of the molecular orbitals of the overall system as well as those of the donor and acceptor orbitals obtained by the partitioning procedure outlined in section IIB. Also shown, in comparison, are the energy levels of the isolated $\left(\mathrm{TiO}_{2}\right)_{24}\left(\mathrm{H}_{2} \mathrm{O}\right)_{30}$ cluster and the isolated chromophore C343. The isolated $\left(\mathrm{TiO}_{2}\right)_{24}\left(\mathrm{H}_{2} \mathrm{O}\right)_{30}$ cluster exhibits a rather dense level structure with a valence and conduction band separated by a band gap. The calculated value for band gap of the finite cluster is 2.8 $\mathrm{eV}$, which is somewhat smaller than calculated $\left(4.0 \mathrm{eV}^{88}\right)$ and experimental $\left(3.4 \mathrm{eV}^{89}\right)$ values for anatase $\mathrm{TiO}_{2}$ nanoparticles. This underestimation of the band gap is presumably due to the added hydrogen and hydroxyl groups used to saturate the cluster. Unsaturated finite clusters ${ }^{88}$ show typically a larger band gap 

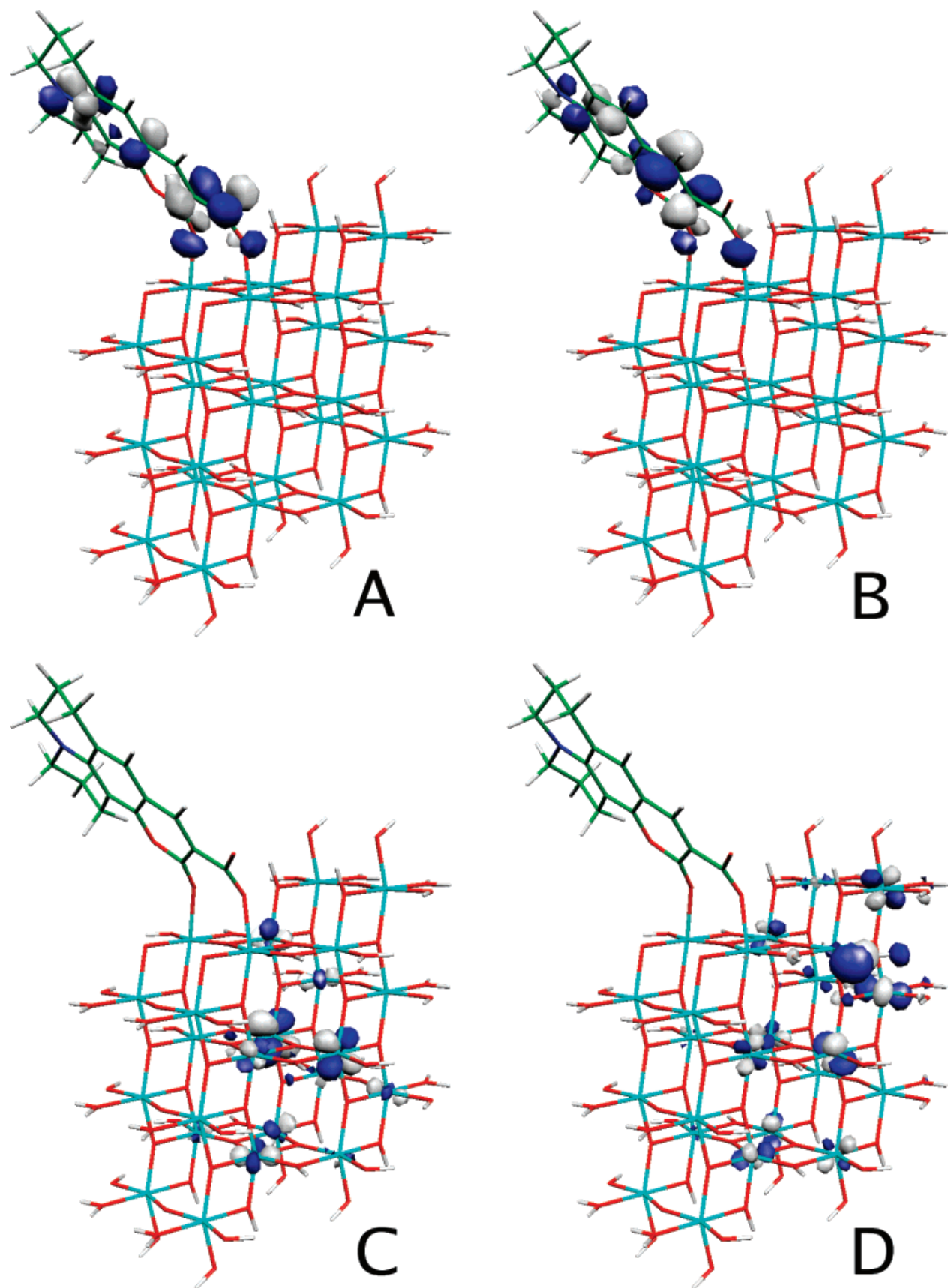

Figure 3. Selected orbitals obtained with the partitioning procedure. Shown are the orbitals corresponding to the HOMO orbital of isolated C343 (A), the donor orbital (B), and two representative acceptor orbitals (C and D).

than bulk anatase. A detailed analysis of the orbitals of $\left(\mathrm{TiO}_{2}\right)_{24}$ $\left(\mathrm{H}_{2} \mathrm{O}\right)_{30}$ revealed that the lowest unoccupied orbitals are localized predominantly on saturation groups (cf. Figure 2, rightmost panel) and can be regarded as levels in the band gap. As has been demonstrated in a computational study of similar clusters, ${ }^{83}$ this deficiency of the saturated cluster model is not expected to have a significant influence on the electronic levels involved in ET transitions. Moreover, these levels in the band gap do not couple to the donor levels of C343 in the complex (see below)

The adsorption of the chromophore $\mathrm{C} 343$ on the $\left(\mathrm{TiO}_{2}\right)_{24-}$ $\left(\mathrm{H}_{2} \mathrm{O}\right)_{30}$ cluster changes the level structure noticeably. In particular, it introduces energy levels in the lower part of the band gap, which can be associated with the highest occupied molecular orbitals (in particular HOMO and HOMO - 2) of the isolated chromophore. The complex also exhibits a few energy levels in the higher part of the band gap, which correspond to orbitals localized at the $\mathrm{TiO}_{2}$ cluster. While the highest occupied levels of $\mathrm{C} 343$ are located in the band gap and thus retain their discrete structure in the complex, the lowest unoccupied levels of C343 (in particular LUMO and LUMO + 1) are located energetically in the conduction band of $\mathrm{TiO}_{2}$. As a consequence, in the complex these levels are dissolved in the dense manifold of conduction band levels.

The partitioning procedure results in orbitals localized at the chromophore and the $\mathrm{TiO}_{2}$ cluster, respectively. Although the energy levels of both parts resemble the level structure of isolated $\mathrm{C} 343$ and $\mathrm{TiO}_{2}$ to some extent, there are noticeable differences due to the fact that these orbitals are not the molecular orbitals of the separated parts but take some of the interaction into account. For the study of electron-injection dynamics, the donor orbital is chosen as the orbital that corresponds in the limit of infinite separation to the LUMO of isolated C343. Employing TD-DFT calculations, it has been 


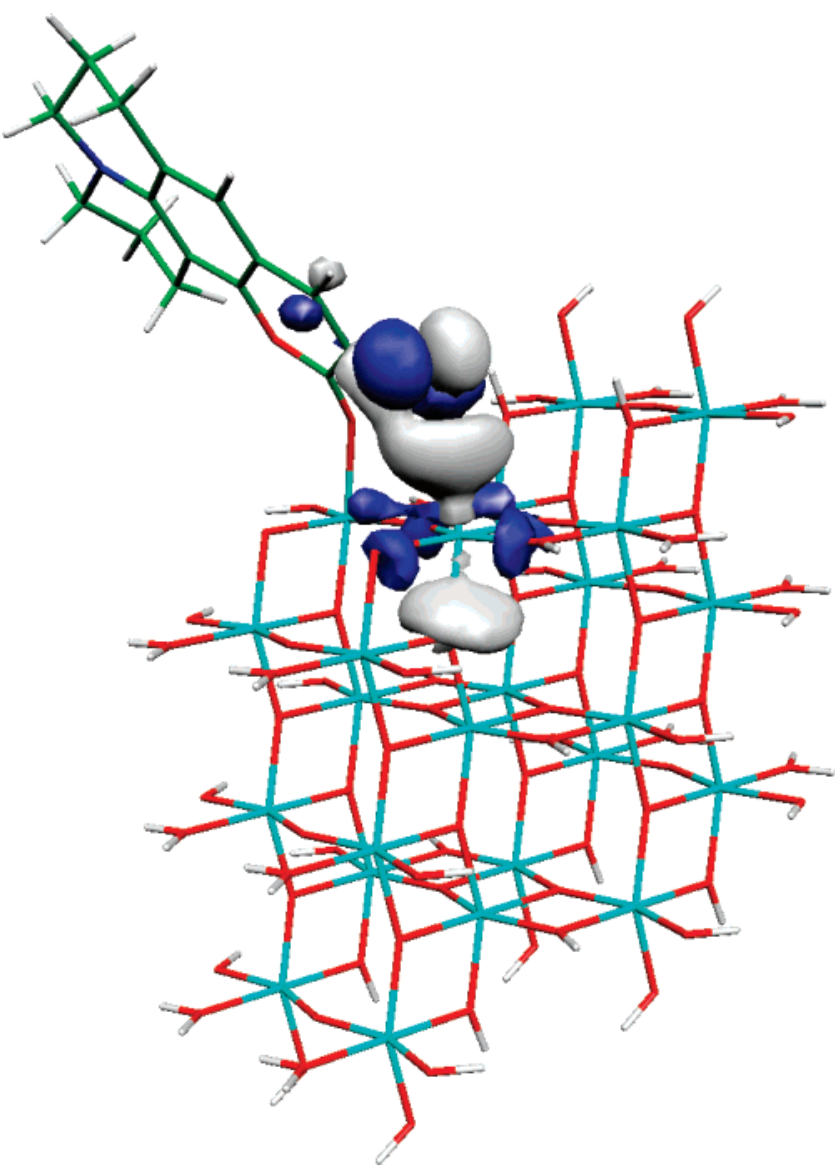

Figure 4. Example of a "donor" orbital, where the orthogonalization procedure results in noticeable contributions at the $\mathrm{TiO}_{2}$ cluster

shown recently ${ }^{42}$ that this orbital is the dominant excitation in isolated C343 and in complexes with small clusters of $\mathrm{TiO}_{2} \cdot{ }^{90}$ The acceptor orbitals include all orbitals localized at the $\mathrm{TiO}_{2}$ cluster with energies above the energy of the HOMO of the overall system.

The local character of the orbitals resulting from the partitioning procedure is illustrated in Figure 3 . The orbital that can be associated to the HOMO orbital of isolated C343 (in the complex, this orbital corresponds to HOMO - 1) as well as the donor orbital are well localized on the chromophore. The acceptor orbitals are, however, localized at the $\mathrm{TiO}_{2}$ cluster. The latter orbitals are dominated by the $3 \mathrm{~d}$ orbitals of titanium.

In the system investigated, the localization works very well for all donor and acceptor orbitals that participate in the ET process. In the energy range of interest, the maximal nonlocal contribution to donor or acceptor states is about $1 \%$. It should be emphasized, however, that the localization method (as any localization procedure) should be tested for each system to which it is applied. In the method used here, problems may arise because due to the orthogonalization, which precedes the donor-acceptor separation, some of the local character of the orbitals may be lost. This concerns in particular orbitals localized close to the dye-semiconductor binding site. An example of such an orbital is shown in Figure 4. Although this orbital is predominantly localized at $\mathrm{C} 343$ it also has non-negligible contributions from the $\mathrm{TiO}_{2}$ cluster. However, this orbital corresponds to an occupied orbital of the overall system, with an energy well separated from the donor state, and is thus not involved in the electron-injection process.

The donor-acceptor coupling matrix elements obtained based on the partitioning are depicted in Figure 5. It is seen that a

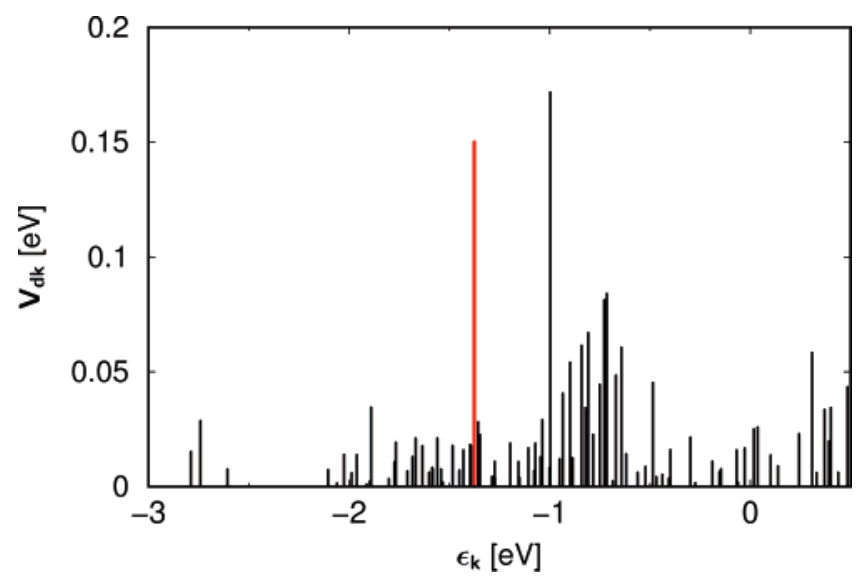

Figure 5. Modulus of the donor-acceptor coupling matrix elements $V_{d k}$. The red line indicates the energy of the donor level, $\epsilon_{d}$.

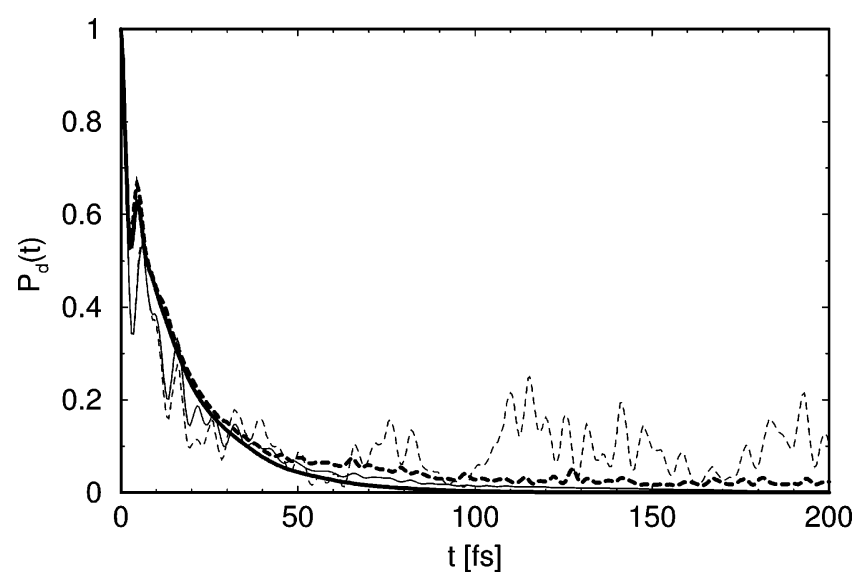

Figure 6. Population dynamics of the donor state after photoexcitation. Shown are results obtained with vibronic coupling (thick lines), without vibronic coupling (thin lines), for the finite $\mathrm{TiO}_{2}$ cluster (dashed lines), and for the model of an infinite $\mathrm{TiO}_{2}$ surface (solid lines).

rather broad distribution of acceptor states couples to the donor state. In contrast to the Newns model used in our previous study, ${ }^{41}$ where the donor-acceptor coupling varies smoothly with the energy $\epsilon_{k}$, the first-principles-based couplings exhibit significant structures.

B. Electron-Transfer Dynamics. On the basis of the firstprinciples model discussed above, we have performed dynamical simulations of the ET dynamics. Thereby, the chosen donor state, all 1184 acceptor states, and 39 modes of C343 (selected according to their electronic-vibrational coupling strength) were taken into account explicitly. The continuous distribution of solvent modes was represented by 20 additional oscillators.

Figure 6 shows the result of the simulation for the population of the donor state after photoexcitation (thick dashed line). The simulation predicts an ultrafast injection of the electron from the donor state localized at the chromophore into the quasicontinuum of acceptor states localized in the $\mathrm{TiO}_{2}$ cluster on a time scale (1/e time) of $\sim 13$ fs. In addition to the ultrafast injection component, the simulation results also exhibit a small component of slower injection dynamics as well as oscillatory structures superimposed on the decay. A comparison with a purely electronic calculation (thin dashed line in Figure 6), where the coupling to the nuclear degrees of freedom has been set to zero, reveals that the high-frequency oscillations are of electronic origin, i.e., can be classified as electronic coherence. In the results of the purely electronic calculation, these coherence effects are much more pronounced than in the full vibronic results (thick dashed line in Figure 6). Thus, the coupling to 


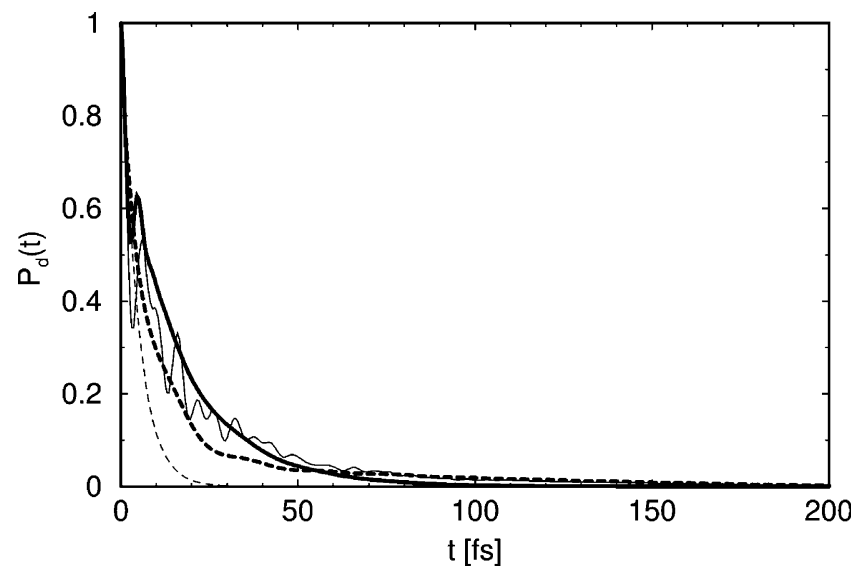

Figure 7. Population dynamics of the donor state after photoexcitation obtained by employing the first-principles-based model (solid lines) and the semiempirical model for the electronic coupling (dashed lines). Fully vibronic and purely electronic population dynamics are depicted by thick lines and thin lines, respectively.

the nuclear degrees of freedom results in a significant decoherence effect. In addition to this quenching of electronic coherence, the coupling to the nuclear degrees of freedom also introduces slower oscillations of vibrational origin. This vibrational coherence effect is, however, small in the present system.

The results in Figure 6 also show that the electron-injection process is not complete: There is a small but noticeable probability $(\sim 2 \%)$ to find the electron for long times at the donor state. This finite probability for electron trapping at the donor also persists for even longer times (data not shown). The incomplete injection is a result of the finite cluster considered. If an additional imaginary part $(\sigma=1 \mathrm{eV})$ is included into the orbital energies of the outer atoms of the $\mathrm{TiO}_{2}$ cluster (cf. the discussion in section IIB and the Appendix) to mimic electron injection at an infinite surface, then the donor population decays to zero. This is demonstrated by the solid lines in Figure 6 . The result for the infinite system agrees well with the one for the finite $\mathrm{TiO}_{2}$ cluster for short times but decays to zero for longer times, corresponding to a complete electron-injection process.

The time scale of electron injection predicted by the simulation $(\sim 13 \mathrm{fs})$ is faster than the experimental results for the $\mathrm{C} 343-\mathrm{TiO}_{2}$ system, where injection times in the range of 20$200 \mathrm{fs}$ have been found using different techniques. . $^{3,5,6,13,81}$ This discrepancy can have a variety of reasons. As discussed above, the electron-injection dynamics are not a single-exponential decay but also exhibit a slower injection component as well as oscillatory structures, which may result effectively in an overall longer injection time. Furthermore, the dynamics depend on the energy-level structure, in particular the relative position of the donor state with respect to the conduction band edge, as well as on the donor-acceptor coupling matrix elements and thus on the accuracy of the electronic structure model used. Another reason for the deviation may be the limited time resolution of the experiments.

Finally, Figure 7 shows a comparison of results obtained from the first-principles model, where the electronic energies and electronic coupling matrix elements have been determined by electronic structure calculations, with results where these parameters have been determined by the semiempirical Newns model. ${ }^{41}$ Overall, the two models predict similar ET characteristics and thus confirm the robustness of the Newns model. The major differences are the slower ultrafast injection component (by about a factor of 2) and the electronic coherences in the result based on the first-principles model. The different ET time scale is caused by the overall stronger donor-acceptor coupling in the semiempirical Newns model. The different importance of electronic coherence effects, however, is related to the way the donor-acceptor coupling is described in the two models. In the semiempirical Newns model the donor-acceptor coupling matrix elements $V_{d k}$ are described by a continuous function $\Gamma(E)$ (cf. the discussion in the Appendix). Because in the model used the function $\Gamma(E)$ varies slowly and, furthermore, the energy of the donor state is well above the conduction band edge of the semiconductor, ${ }^{41}$ the result of the Newns model does not show electronic coherence effects. In the first-principles model, however, the donor-acceptor coupling matrix elements $V_{d k}$ depend significantly on the energy $\epsilon_{k}$, thus resulting in pronounced electronic coherence effects in the population dynamics. The comparison in Figure 7 also shows that the effect of the coupling to the nuclear degrees of freedom on the electron-injection time scale is more pronounced in the semiempirical Newns model.

\section{Concluding Remarks}

In this paper, we have outlined a first-principles-based method to describe the quantum dynamics of heterogeneous ET reactions at dye-semiconductor interfaces. The method employs an expansion of the Hamiltonian in a diabatic basis of electronic donor and acceptor states localized at the dye molecule and the semiconductor substrate, respectively. To determine the diabatic states and thus the electronic energies and the donor-acceptor coupling matrix elements, we have used a partitioning scheme. This scheme is based on a partitioning of the overall Hilbert space into a donor and acceptor part and a subsequent separate diagonalization of the two blocks of the Hamiltonian to define the donor and acceptor states. In this article, we have employed the Kohn-Sham orbitals and matrix to describe the system and thus used an effective single-electron Hamiltonian. The extension of the method to many-electron states will be the subject of future work.

As an application, we have considered photoinduced electron injection for coumarin 343 adsorbed at titanium oxide nanoparticles. The latter were modeled by a finite titanium oxide cluster. The quantum dynamics of the ET reaction were simulated using the ML-MCTDH method, which allows an efficient and accurate description of this system, which comprises many electronic states and nuclear degrees of freedom. The results demonstrate the ultrafast character of the electroninjection process in this system. The ET dynamics exhibit significant electronic coherence effects. The coupling to the nuclear degrees of freedom of the chromophore results in a quenching of the coherence effects.

In the present application we have assumed that the photoexcitation by an ultrashort laser pulse can be described by an instantaneous transition from the electronic ground state to the donor state. As shown previously, ${ }^{72}$ the methodology also allows us to include the laser field explicitly in the calculation to study the influence of the laser pulse on the dynamics of these ultrafast ET reactions. Employing, furthermore, the nonperturbative description of matter-field interaction outlined recently, ${ }^{80,91}$ it will also be possible to simulate time-resolved nonlinear spectra, which will allow a direct comparison with experimental results.

\section{Appendix}

Description of Electron Injection for a Chromophore Adsorbed on an Extended Substrate. As mentioned above, 
the partitioning procedure introduced in section IIB is not limited to dye-semiconductor systems with a finite semiconductor cluster but can, in principle, also be applied to a dye molecule adsorbed on an extended surface. One possibility is to employ the slab model and electronic structure calculations with periodic boundary conditions..$^{92}$ Alternatively, the effect of an infinite semiconductor substrate can also be described using surface Green's function techniques. ${ }^{59}$ In this appendix, we outline an approximate variant of the latter method.

To this end, let us assume that the partitioning procedure outlined in section IIB has been performed for a chromophore adsorbed on an infinite surface (e.g., using the slab model) resulting in a Hamiltonian of the form of eq 2.1. We first show that if one is only interested in observables that are localized on the chromophore, then the influence of the semiconductor substrate and the chromophore surface coupling on the dynamics is fully characterized by the function

$$
\Gamma(t)=\sum_{k}\left|V_{d k}\right|^{2} \mathrm{e}^{-\mathrm{i} \epsilon_{k} t}
$$

or, equivalently, its Fourier transform

$$
\Gamma(E)=2 \pi \sum_{k}\left|V_{d k}\right|^{2} \delta\left(E-\epsilon_{k}\right)
$$

The latter function describes the density of states of the semiconductor substrate weighted by the donor-acceptor coupling and is also called the energy-dependent decay width of the donor state.

To demonstrate this fact, let us consider the electronic projection of the time-dependent vibronic wave function

$$
|\Psi(t)\rangle=\mathrm{e}^{-\mathrm{i} H t}\left|\Psi_{0}\right\rangle
$$

onto the donor state

$$
\left\langle\psi_{d} \mid \Psi(t)\right\rangle=\left\langle\psi_{d}\left|\mathrm{e}^{-\mathrm{i} H t}\right| \Psi_{0}\right\rangle
$$

where $\left|\Psi_{0}\right\rangle$ denotes the initial state and the Hamiltonian $H$ is given by eq 2.15. In the following, we will assume that the initial wave function is localized at the chromophore, i.e.,

$$
\left\langle\psi_{k} \mid \Psi_{0}\right\rangle=0
$$

Using projection-operator techniques, it is straightforward to show $^{53}$ that the equation of motion for $\left\langle\psi_{d} \mid \Psi(t)\right\rangle$ is given by

$$
\begin{aligned}
& \frac{\partial}{\partial t}\left\langle\psi_{d} \mid \Psi(t)\right\rangle= \\
& \quad-i H_{N_{d}}\left\langle\psi_{d} \mid \Psi(t)\right\rangle-\int_{0}^{t} \mathrm{~d} \tau \Gamma(\tau) \mathrm{e}^{-i H_{\mathrm{N} a} \tau}\left\langle\psi_{d} \mid \Psi(t-\tau)\right\rangle
\end{aligned}
$$

where

$$
\begin{aligned}
H_{\mathrm{N}_{d}} & =\left\langle\psi_{d}|H| \psi_{d}\right\rangle \\
& =\epsilon_{d}+\frac{1}{2} \sum_{l}\left(P_{l}^{2}+\Omega_{l}^{2} Q_{l}^{2}\right)+\sum_{l} \kappa_{l}^{d} Q_{l}+ \\
& \frac{1}{2} \sum_{j}\left(p_{j}^{2}+\omega_{j}^{2} x_{j}^{2}\right)+\sum_{j} c_{j}^{d} x_{j}
\end{aligned}
$$

denotes the nuclear Hamiltonian in the donor state and

$$
\begin{aligned}
H_{\mathrm{N}_{a}}=\frac{1}{2} \sum_{l}( & \left.P_{l}^{2}+\Omega_{l}^{2} Q_{l}^{2}\right)+ \\
& \sum_{l} \kappa_{l}^{a} Q_{l}+\frac{1}{2} \sum_{j}\left(p_{j}^{2}+\omega_{j}^{2} x_{j}^{2}\right)+\sum_{j} c_{j}^{a} x_{j}
\end{aligned}
$$

is the nuclear Hamiltonian in the acceptor states. Equation A.6 shows that the influence of the semiconductor substrate and of the chromophore-surface coupling enters only through the function $\Gamma(t)$ or, equivalently, its Fourier transform $\Gamma(E)$.

While $\Gamma(t)$ is a smooth function for finite semiconductor clusters and extended surfaces, $\Gamma(E)$ is a continuous function only for a chromophore adsorbed on an infinite surface. The width function $\Gamma(E)$ is the imaginary part of the self-energy in the donor state $\Sigma_{d d}(E)$

$$
\begin{gathered}
\Gamma(E)=-2 \operatorname{Im} \Sigma_{d d}(E) \\
\Sigma_{d d}(E)=\left\langle\psi_{d}\left|V G_{\mathrm{sf}}(E) V\right| \psi_{d}\right\rangle
\end{gathered}
$$

where

$$
V=\sum_{k}\left(\left|\psi_{d}\right\rangle V_{d k}\left\langle\psi_{k}|+| \psi_{k}\right\rangle V_{k d}\left\langle\psi_{d}\right|\right)
$$

describes the interaction between the chromophore and the semiconductor surface and

$$
G_{\mathrm{sf}}(E)=\left(E+\mathrm{i} \eta-H_{\mathrm{sf}}\right)^{-1}
$$

is the electronic Green's function of the semiconductor surface. Thereby, $H_{\text {sf }}$ denotes the electronic Hamiltonian of the semiconductor substrate and $\eta$ is the usual positive infinitesimal.

In our previous work on heterogeneous $\mathrm{ET}^{37,41}$ we have used a semiempirical Newns model, where $\Gamma(E)$ was parametrized based on a tight-binding model developed by Petersson et al. ${ }^{43}$ The parameters within this model have been determined to fit the lower $3 \mathrm{~d}$ group of the $\mathrm{TiO}_{2}$ conduction band (see refs 41 and 43 for further details).

As was mentioned above, a first-principles calculation of $\Gamma(E)$ is possible by employing the slab model and electronic structure calculations with periodic boundary conditions. Alternatively, a finite part of the semiconductor surface (in the following referred to as the cluster) can be treated explicitly, and the influence of the remaining infinite part can be described using surface Green's function techniques. ${ }^{59}$ Within this method the effect of the infinite substrate enters via a self-energy matrix that is localized at the boundary of the cluster, which is treated explicitly. To discuss this approach, we introduce a projection operator $P$ that projects on the cluster, i.e., on the part of the substrate that is explicitly treated. Using standard projectionoperator techniques one obtains for the projected Green's function of the surface

$$
P G_{\mathrm{sf}}(E) P=\left(E+\mathrm{i} \eta-P H_{\mathrm{sf}} P-M(E)\right)^{-1}
$$

Here, $M(E)$ is a self-energy term that describes the influence of the remainder of the substrate given by

$$
M(E)=P H_{\mathrm{sf}} Q\left(E+\mathrm{i} \eta-Q H_{\mathrm{sf}} Q\right)^{-1} Q H_{\mathrm{sf}} P
$$

where $Q=1-P$. The self-energy $M(E)$ in eq A.13 should not be confused with the self-energy $\Sigma(E)$ defined in eq A.9b. While the latter describes the influence of the overall substrate on the adsorbate, the former describes the effect of the remaining part of the infinite substrate, without the explicitly treated $\mathrm{TiO}_{2}$ 
cluster, on the explicitly treated system, i.e., the complex of the dye molecule and the finite $\mathrm{TiO}_{2}$ cluster.

Within our treatment outlined in section IIB the projection operator $P$ is given by the orthogonal orbitals of the explicitly treated acceptor space

$$
P=\sum_{n}\left|\tilde{\varphi}_{n}^{a}\right\rangle\left\langle\tilde{\varphi}_{n}^{a}\right|
$$

and the projected Hamiltonian of the substrate, $P H_{\mathrm{sf}} P$, is given by the Fock matrix of the acceptor space in the orthogonal basis

$$
\left\langle\tilde{\varphi}_{n}^{a}\left|P H_{\mathrm{sf}} P\right| \tilde{\varphi}_{m}^{a}\right\rangle=\tilde{F}_{a a, n m}
$$

In principle, the energy-dependent self-energy matrix $M(E)$ can be calculated using surface Green's function techniques. In the application considered in this paper, we have used a variant of this method, which approximates the self-energy by a constant diagonal imaginary part $\sigma$ at the outer atoms of the explicitly treated $\mathrm{TiO}_{2}$ cluster; i.e., $M(E) \approx M$ with $M_{j j}=-i \sigma / 2$ if $j$ belongs to an orbital of the outer atoms of the $\mathrm{TiO}_{2}$ cluster, and $M_{j k}=0$ otherwise. Thus, within this method the acceptor block of the Fock matrix from eq 2.5 is replaced by

$$
\tilde{F}_{a a} \rightarrow \tilde{F}_{a a}+M
$$

As a result, the Fock matrix is no longer real but complex symmetric.

In our practical implementation, we calculate $\Gamma(E)$ via the relation to the self-energy $\Sigma_{d d}(E)$, eq A.9a, with

$$
\begin{aligned}
\Sigma_{d d} & =\left\langle\psi_{d}\left|V G_{\mathrm{sf}}(E) V\right| \psi_{d}\right\rangle \\
& =\left\langle\psi_{d}\left|V P G_{\mathrm{sf}}(E) P V\right| \psi_{d}\right\rangle \\
& =\tilde{F}_{d a}^{\prime} G_{a a}\left(\tilde{F}_{d a}^{\prime}\right)^{\dagger}
\end{aligned}
$$

Here, $G_{a a}$ denotes the matrix representation of $P G_{\mathrm{sf}}(E) P$ in the orthogonal basis $\left|\tilde{\varphi}_{n}^{a}\right\rangle$

$$
G_{a a, n m}=\left\langle\tilde{\varphi}_{n}^{a}\left|P G_{\mathrm{sf}}(E) P\right| \tilde{\varphi}_{m}^{a}\right\rangle
$$

The matrix $\tilde{F}_{d a}^{\prime}$ is obtained by diagonalizing the donor block $\tilde{F}_{d d}$ according to eq 2.7 and transforming the off-diagonal blocks according to $\tilde{F}_{d a}^{\prime}=D_{d}^{\dagger} \tilde{F}_{d a}$. Furthermore, in eq A.17, we have assumed that $Q V\left|\psi_{d}\right\rangle=0$, which is expected to be a very good approximation for the size of the $\mathrm{TiO}_{2}$ cluster employed in the present study.

To determine $G_{a a}$, we calculate the right eigenvectors and eigenvalues of the complex symmetric matrix $\tilde{F}_{a a}+M$. Denoting the matrix of right eigenvectors by $U$ and the diagonal matrix of eigenvalues by $\lambda$ and using the identities $\tilde{F}_{d a}^{\prime \dagger}=\tilde{F}_{d a}^{\prime}$ and $U W^{-1} U^{\mathrm{T}}=1$, where $W=U^{\mathrm{T}} U$ is the overlap of the eigenvectors of $\tilde{F}_{a a}+M$, we obtain the energy-dependent decay width for the selected donor state

$$
\Gamma(E)=-2 \operatorname{Im}\left\{\tilde{F}_{d a}^{\prime} U \frac{1}{W(E-\lambda)} U^{\mathrm{T}} \tilde{F}_{a d}^{\prime}\right\}
$$

Alternatively, the Green's function $G_{a a}$ could also be determined directly for each energy $E$ using, e.g., matrix inversion. However, in the present context, the diagonalization procedure employed is more advantageous because it has to be performed only once.

In the dynamical simulations, the continuum of electronic states implicit in the continuous function $\Gamma(E)$ is discretized and represented by a finite number of states $\left|\psi_{k}\right\rangle$ employing eq
A. 2 as described previously. ${ }^{37}$ In the present applications, 6000 electronic states within an equidistant discretization schema were sufficient to represent the electronic continuum over the time scale of interest.

Acknowledgment. We thank Wolfgang Domcke for numerous helpful discussions. The generous allocation of computing time by the National Energy Research Scientific Computing Center and the Leibniz Rechenzentrum, Munich, is gratefully acknowledged. This work has been supported by the Deutsche Forschungsgemeinschaft (DFG) through the DFG-Cluster of Excellence Munich-Centre for Advanced Photonics and a research grant (M.T.), the Fonds der Chemischen Industrie (MT), the National Science Foundation CAREER award CHE-0348956 (H.W.), the Alexander von Humboldt foundation, and research grant GACR 202/07/0833 (M.C.).

\section{References and Notes}

(1) Moser, J. E.; Grätzel, M. Chem. Phys. 1993, 176, 493

(2) Hagfeldt, A.; Grätzel, M. Chem. Rev. 1995, 95, 49.

(3) Rehm, J. M.; McLendon, G. L.; Nagasawa, Y.; Yoshihara, K.; Moser, J.; Grätzel, M. J. Phys. Chem. 1996, 100, 9577.

(4) Martini, I.; Odak, J.; Hartland, G. V.; Kamat, P. V. J. Chem. Phys. 1997, 107, 8064 .

(5) Gosh, H. N.; Asbury, J. B.; Wang, Y.; Lian, T. J. Phys. Chem. B 1998, 102, 10208

(6) Wachtveitl, J.; Huber, R.; Spörlein, S.; Moser, J.; Grätzel, M. Int. J. Photoenergy 1999, 1, 153.

(7) Willig, F.; Zimmermann, C.; Ramakrishna, S.; Storck, W. Electrochim. Acta 2000, 45, 4565.

(8) Zimmermann, C.; Willig, F.; Ramakrishna, S.; Burfeindt, B.; Pettinger, B. J. Phys. Chem. B 2001, 105, 9245.

(9) Asbury, J.; Hao, E.; Wang, Y.; Ghosh, H. N.; Lian, T. J. Phys. Chem. B 2001, 105, 4545.

(10) Ramakrishna, G.; Gosh, H. N. J. Phys. Chem. A 2002, 106, 2545.

(11) Schnadt, J.; Brühwiler, P. A.; Patthey, L.; O’Shea, J. N.; Södergreen,

S.; Odellus, M.; Ahuja, R.; Karis, O.; Bässler, M.; Persson, P.; Siegbahn, H.; Lunell, S.; Martenson, N. Nature 2002, 418, 620.

(12) Walters, K. A.; Gaal, D. A.; Hupp, J. T. J. Phys. Chem. B 2002, 106,5139

(13) Huber, R.; Moser, J. E.; Grätzel, M.; Wachtveitl, J. Chem. Phys 2002, 285, 39 .

(14) Huber, R.; Moser, J. E.; Grätzel, M.; Wachtveitl, J. J. Phys. Chem. B 2002, 106, 6494

(15) Kallioinen, J.; Benkö, G.; Sundström, V.; Korrpi-Tommola, J.; Yartsev, A. J. Phys. Chem. B 2002, 106, 4396.

(16) Takeshita, K.; Sasaki, Y.; Kobashi, M.; Tanaka, Y.; Maeda, S. Yamakata, A.; Ishibashi, T.; Onishi, H. J. Phys. Chem. B 2003, 107, 4156. (17) Wang, L.; Ernstorfer, R.; Willig, F.; May, V. J. Phys. Chem. B 2005, 109, 9589.

(18) Hagfeldt, A.; Grätzel, M. Acc. Chem. Res. 2000, 33, 269

(19) Grätzel, M. Nature 2001, 414, 338.

(20) Benkö, G.; Kallioinen, J.; Korppi-Tommola, J.; Yartsev, A.; Sundström, V. J. Am. Chem. Soc. 2002, 124, 489.

(21) Stier, W.; Prezhdo, O. Isr. J. Chem. 2002, 42, 213.

(22) Stier, W.; Prezhdo, O. J. Phys. Chem. B 2002, 106, 8047.

(23) Stier, W.; Prezhdo, O. Adv. Mater. 2004, 16, 240.

(24) Duncan, W.; Stier, W.; Prezhdo, O. J. Am. Chem. Soc. 2005, 127, 7941.

(25) Duncan, W.; Stier, W.; Prezhdo, O. J. Phys. Chem. B 2005, 109 , 365

(26) Rego, L.; Batista, V. J. Chem. Phys. 2005, 122, 154709.

(27) Newns, D. Phys. Rev. 1969, 178, 1123

(28) Ramakrishna, S.; Willig, F.; May, V. Phys. Rev. B 2000, 62, 16330

(29) Ramakrishna, S.; Willig, F. J. Phys. Chem. B 2000, 104, 68. 2743

(31) Schreiber, M.; Kondov, I.; Kleinekathöfer, U. J. Lumin. 2001, 94 471.

(32) Ramakrishna, S.; Willig, F.; May, V. Chem. Phys. Lett. 2002, 351, 242.

(33) Ramakrishna, S.; Willig, F.; May, V. J. Phys. Chem. B 2003, 107, 607.

(34) Wang, L.; May, V. J. Chem. Phys. 2004, 121, 8039.

(35) Liang, K.; Lin, C.-K.; Chang, H.-C.; Lin, S. J. Chem. Phys. 2006, $125,154706$.

(36) Wang, L.; Willig, F.; May, V. J. Chem. Phys. 2006, 124, 014712

(37) Thoss, M.; Kondov, I.; Wang, H. Chem. Phys. 2004, 304, 169. 

2979.

(38) Wang, H.; Thoss, M.; Miller, W. H. J. Chem. Phys. 2001, 115,

(39) Wang, H.; Thoss, M. Isr. J. Chem. 2002, 42, 167.

(40) Wang, H.; Thoss, M. J. Chem. Phys. 2003, 119, 1289.

(41) Kondov, I.; Thoss, M.; Wang, H. J. Phys. Chem. A 2006, 110, 1364.

(42) Kondov, I.; Wang, H.; Thoss, M. Int. J. Quantum Chem. 2006, $106,1291$.

(43) Petersson, A.; Ratner, M.; Karlsson, H. J. Phys. Chem. B 2000, 104,8498 .

(44) Mulliken, R. J. Am. Chem. Soc. 1952, 74, 811.

(45) Hush, N. Prog. Inorg. Chem. 1968, 13, 1005.

(46) Cave, R.; Newton, M. Chem. Phys. Lett. 1996, 249, 15.

(47) Cave, R.; Newton, M. J. Chem. Phys. 1997, 106, 9213.

(48) Voityuk, A.; Rösch, N. J. Chem. Phys. 2000, 117, 5607.

(49) For an overview, see, for example, ref 93 and references therein.

(50) Feshbach, H. Ann. Phys. 1962, 19, 287.

(51) O'Malley, T. Phys. Rev. 1967, 162, 98.

(52) Domcke, W. Phys. Rev. A 1983, 28, 2777.

(53) Domcke, W. Phys. Rep. 1991, $208,97$.

(54) Similar partitioning methods have been used in variety of other fields, for example, in the context of electron transport through single molecule junctions. ${ }^{94}$ Particularly closely related to the approach used here is the method employed in ref 60 .

(55) Kurnikov, L.; Beratan, D. J. Chem. Phys. 1996, 105, 9561.

(56) Galperin, M.; Toledo, S.; Nitzan, A. J. Chem. Phys. 2002, 117, 10817

(57) Löwdin, P.-O. J. Chem. Phys. 1950, 18, 365.

(58) Mayer, I. Int. J. Quantum Chem. 2002, 90, 63.

(59) Xue, Y.; Ratner, M. Phys. Rev. B 2001, 68, 115406.

(60) Benesch, C.; Cizek, M.; Thoss, M.; Domcke, W. Chem. Phys. Lett. 2006, 430,355 .

(61) This correponds to the Condon approximation, which is often employed in ET systems, and is expected to be valid for the relatively rigid systems considered here.

(62) Dushinski, F.; Sassetti, M.; Weiss, U. Acta Physicochim. URSS 1937, 7, 551 .

(63) Myers, A. Chem. Rev. 1996, 96, 911.

(64) Conical Intersections: Electronic Structure, Dynamics and Spectroscopy; Domcke, W., Yarkony, D. R., Köppel, H., Eds.; World Scientific: Singapore, 2004.

(65) Marcus, R. A.; Sutin, N. Biochim. Biophys. Acta 1985, 811, 265.

(66) Weiss, U. Quantum Dissipative Systems, 2nd ed.; World Scientific: Singapore, 1999.

(67) Makri, N. J. Phys. Chem. B 1999, 103, 2823.

(68) Georgievskii, Y.; Hsu, C.-P.; Marcus, R. A. J. Chem. Phys. 1999, $110,5307$.

(69) Rips, I.; Jortner, J. J. Chem. Phys. 1987, 87, 2090.

(70) May, V.; Kühn, O. Charge and Energy Transfer Dynamics in Molecular Systems: A Theoretical Introduction; Wiley-VCH: Berlin, 2000.

(71) Jimenez, R.; Fleming, G. R.; Kumar, P. V.; Maroncelli, M. Nature 1994, 369, 471 .

(72) Wang, H.; Thoss, M. J. Chem. Phys. 2006, 124, 034114.

(73) Meyer, H.-D.; Manthe, U.; Cederbaum, L. S. Chem. Phys. Lett. $1990,165,73$.

(74) Manthe, U.; Meyer, H.-D.; Cederbaum, L. S. J. Chem. Phys. 1992, $97,3199$.
(75) Beck, M. H.; Jäckle, A.; Worth, G. A.; Meyer, H.-D. Phys. Rep. 2000, 324, 1 .

(76) Meyer, H.-D.; Worth, G. A. Theor. Chem. Acc. 2003, 109, 251.

(77) Thoss, M.; Wang, H. Chem. Phys. 2006, 322, 210.

(78) Wang, H.; Thoss, M. J. Phys. Chem. A 2003, 107, 2126.

(79) Thoss, M.; Domcke, W.; Wang, H. Chem. Phys. 2004, 296, 217.

(80) Wang, H.; Thoss, M. Chem. Phys. Lett. 2004, 389, 43.

(81) Murakoshi, K.; Yanagida, S.; Capel, M.; Castner, E. Interfacial electron transfer dynamics of photosensitized zinc oxide nanoclusters. In Nanostructured Materials: Clusters, Composites, and Thin Films; Chalaev, V. M., Moskovits, M.. Eds.; ACS Symposium Series 679; American Chemical Society: Washigton, DC, 1997; pp 221-238.

(82) Burdett, J. K.; Hughbanks, T.; Miller, G. J.; Richardson, J. W., Jr.; Smith, J. V. J. Am. Chem. Soc. 1987, 109, 3639.

(83) Bredow, T.; Jug, K. J. Phys. Chem. 1995, 99, 285.

(84) Redfern, P. C.; Zapol, P.; Curtiss, L. A.; Rajh, T.; Thurnauer, M. C. J. Phys. Chem. B 2003, 107, 11419.

(85) Kondov, I.; Markmann, A.; Thoss, M., to be submitted for publication.

(86) Frisch, M. J.; Trucks, G. W.; Schlegel, H. B.; Scuseria, G. E.; Robb, M. A.; Cheeseman, J. R.; Montgomery, J. A., Jr.; Vreven, T.; Kudin, K. N.; Burant, J. C.; Millam, J. M.; Iyengar, S. S.; Tomasi, J.; Barone, V. Mennucci, B.; Cossi, M.; Scalmani, G.; Rega, N.; Petersson, G. A.; Nakatsuji, H.; Hada, M.; Ehara, M.; Toyota, K.; Fukuda, R.; Hasegawa, J.; Ishida, M.; Nakajima, T.; Honda, Y.; Kitao, O.; Nakai, H.; Klene, M.; Li, X.; Knox, J. E.; Hratchian, H. P.; Cross, J. B.; Bakken, V.; Adamo, C.; Jaramillo, J.; Gomperts, R.; Stratmann, R. E.; Yazyev, O.; Austin, A. J.; Cammi, R.; Pomelli, C.; Ochterski, J. W.; Ayala, P. Y.; Morokuma, K.; Voth, G. A.; Salvador, P.; Dannenberg, J. J.; Zakrzewski, V. G.; Dapprich, S.; Daniels, A. D.; Strain, M. C.; Farkas, O.; Malick, D. K.; Rabuck, A. D.; Raghavachari, K.; Foresman, J. B.; Ortiz, J. V.; Cui, Q.; Baboul, A. G.; Clifford, S.; Cioslowski, J.; Stefanov, B. B.; Liu, G.; Liashenko, A.; Piskorz, P.; Komaromi, I.; Martin, R. L.; Fox, D. J.; Keith, T.; Al-Laham, M. A.; Peng, C. Y.; Nanayakkara, A.; Challacombe, M. Gill, P. M. W.; Johnson, B.; Chen, W.; Wong, M. W.; Gonzalez, C.; Pople, J. A. Gaussian 03, revision B.04; Gaussian, Inc.: Wallingford, CT, 2003.

(87) Ahlrichs, R.; Bär, M.; Häser, M.; Horn, H.; Kölmel, C. Chem. Phys. Lett. 1989, 162, 165 .

(88) Nilsing, M.; Lunell, S.; Persson, P.; Ojamäe, L. Surf. Sci. 2005, 582,49

(89) Kormann, C.; Bahnemann, D.; Hoffmann, M. J. Phys. Chem. 1988, $92,5196$.

(90) It is also noted that the first exited state of isolated C343 corresponds almost exclusively to a HOMO-LUMO excitation. The excitation energy of $3.43 \mathrm{eV}$ (employing TD-DFT at the B3LYP/SV(P) level) is in good agreement with the HOMO-LUMO gap (3.59 eV). Therefore, the use of the LUMO as a model for the excited state is well justified in this system.

(91) Seidner, L.; Stock, G.; Domcke, W. Chem. Phys. Lett. 1994, 228, 665.

(92) Nilsing, M.; Persson, P.; Ojamäe, L. Chem. Phys. Lett. 2005, 415, 375

(93) Köppel, H. In Conical Intersections: Electronic Structure, Dynamics and Spectroscopy; Domcke, W., Yarkony, D. R., H. Köppel, Eds.; World Scientific: Singapore, 2004.

(94) Mujica, V.; Kemp, M.; Ratner, M. J. Chem. Phys. 1994, 101, 6849. 